Published in final edited form as:

Environ Int. 2018 July ; 116: 116-121. doi:10.1016/j.envint.2018.04.007.

\title{
Prenatal exposure to polychlorinated biphenyls and fetal growth in British girls
}

\author{
Jill F. Patela ${ }^{a, b}$, Terryl J. Hartman ${ }^{a, b, c,},{ }^{,}$Andreas Sjodin ${ }^{a}$, Kate Northstone ${ }^{d, e}$, and Ethel V. \\ Taylor ${ }^{\mathrm{a}}$ \\ ${ }^{a}$ Centers for Disease Control and Prevention, Atlanta, GA 30341, United States \\ bDepartment of Epidemiology, Rollins School of Public Health, Emory University, Atlanta, GA \\ 30322, United States \\ 'Winship Cancer Institute, Emory University, Atlanta, GA 30322, United States \\ dThe National Institute for Health Research Collaboration for Leadership in Applied Health \\ Research and Care West (NIHR CLAHRC West) at University Hospitals Bristol NHS Foundation \\ Trust, UK \\ eSchool of Social and Community Medicine, University of Bristol, Bristol, UK
}

\section{Abstract}

Polychlorinated biphenyls (PCBs) are synthetic chemicals that bioaccumulate in the food chain. PCBs were used primarily for industrial applications due to their insulating and fire retardant properties, but were banned in the 1970s in the United States and in the 1980s in the United Kingdom, as adverse health effects following exposure were identified. Previous studies of populations with high PCB exposure have reported inverse associations with birth weight and gestational length. Birth weight is a powerful predictor of infant survival, and low birth weight can predispose infants to chronic conditions in adult life such as diabetes and cardiovascular diseases.

Using data from the Avon Longitudinal Study of Parents and Children, we investigated the association between prenatal exposure to PCBs and fetal growth in a sample of 448 motherdaughter dyads. Concentrations of three common PCB analytes, PCB-118, PCB-153 and PCB-187, were measured in maternal serum collected during pregnancy, and fetal growth was measured by birth weight and birth length. Multivariable linear regression was used to examine the associations between PCB analytes and measures of fetal growth, after adjusting for parity, maternal age, pre-pregnancy BMI, educational status, tobacco use and gestational age of infant at sample collection. Birth length, ponderal index and gestational age were not associated with any of the PCB analytes. Mothers' educational status modified associations for PCB analytes with

\footnotetext{
* Corresponding author at: Rollins School of Public Health, Winship Cancer Institute, Emory University, 1518 Clifton RD NE, CNR 3035, Atlanta, GA 30322, United States. tjhartm@emory.edu (T.J. Hartman).

Author disclosure statement

The authors declare they have no actual or potential competing financial interests.

Disclaimer

The findings and conclusions do not necessarily represent views of the CDC, the NHS, the NIHR or the Department of Health. Use of trade names is for identification only and does not imply endorsement by the CDC, the Public Health Service, or the U.S. Department of Health and Human Services.
} 
birthweight. We observed significant inverse associations with birth weight only among daughters of mothers with less education. Daughter's birth weight was $-138.4 \mathrm{~g}$ lower (95\% CI: -218.0 , -58.9) for each $10 \mathrm{ng} / \mathrm{g}$ lipid increase in maternal serum PCB-118. Similarly, every $10 \mathrm{ng} / \mathrm{g}$ lipid increase in maternal serum PCB-153 was associated with a $-41.9 \mathrm{~g}$ (95\% CI: $-71.6,-12.2)$ lower birth weight. Every $10 \mathrm{ng} / \mathrm{g}$ lipids increase in maternal serum PCB-187, was associated with a $-170.4 \mathrm{~g}(95 \% \mathrm{CI}:-306.1,-34.7)$ lower birth weight, among girls with mothers in the lowest education group.

Our findings suggest that prenatal exposure to PCBs is inversely associated with daughters' birth weight and that mothers' education, which is a possible marker for socioeconomic status, significantly modified the association between maternal PCB concentrations and birth weight in female newborns.

\section{Keywords}

Polychlorinated biphenyls; Endocrine disrupting chemicals; Pregnancy; Birthweight

\section{Introduction}

Polychlorinated biphenyls (PCBs) are a family of synthetic organic chemicals, comprising 209 chemically related compounds that were used between 1930 and 1977 for various industrial applications because of their insulating and fire-retardant properties (Ross, 2004). PCBs were banned in the 1970s in the United States and in the 1980s in the United Kingdom, as adverse health effects following exposure were identified. PCBs are biphenyls with between one and ten chlorine atoms attached, and the degree of chlorination determines the stability and lipophilicity of the specific PCB analyte (Kezios et al., 2012). PCBs with lower degrees of chlorination tend to be more rapidly excreted from the body, while more chlorinated PCB compounds (e.g., PCB 153) are retained for a longer period of time, many with biological half-lives in the order of years (Taylor et al., 1989; El Majidi et al., 2012). There are no known natural sources of PCBs (Public Health, 2000) and once PCBs are released into the environment, they do not readily break down and can easily cycle between air, water and soil. Furthermore, they can be carried long distances and have been found in areas of snow and sea water far from the original release site (Taylor et al., 1989).

The most common sources of environmental exposure to these substances are dairy products, meat and especially fish (Koopman-Esseboom et al., 1994). PCBs are stored mainly in human adipose tissue, and their poor metabolism results in elimination half-lives of approximately 10-15 years (Patandin et al., 1998; Ritter et al., 2011). Animal and human studies have shown that PCBs cross the placenta (Kezios et al., 2012) and the quantities of PCBs found in cord serum may be considerable relative to the size of the developing fetus (Jacobson et al., 1984). Endocrine pathways that are important for fetal development, such as thyroid hormone signaling, can be disrupted by PCBs, potentially leading to decreased in utero growth (Public Health, 2000).

Birth weight is one of the most, if not the most, powerful predictors of infant survival (WHO, 1992), with low birth weight contributing to about 9.1 million infant deaths each 
year. Globally, 17\% of total births are to low birth weight newborns (WHO, 1992). Low birth weight in early childhood can be associated with adiposity in adolescents and earlier pubertal maturation, and is a strong predictor of the development of obesity, hypertension and cardiovascular disease in adults (Wohlfahrt-Veje et al., 2014). Additionally, low birth weight is a predictor for other adverse outcomes such as poor school performance, high blood pressure and cardiovascular diseases (Law et al., 2002; Barker, 2002; Barker, 1998; Thompson et al., 2001). Therefore, it is important to determine whether prenatal PCB exposure is associated with birth outcomes such as low birth weight. The objective of this study was to investigate the association between prenatal exposure to PCBs and fetal growth in a well-characterized British sample of mother-daughter dyads.

\section{Methods}

\subsection{Population}

The Avon Longitudinal Study of Parents and Children (ALSPAC) is a prospective birthcohort study designed to evaluate the influences of genetic and environmental factors on health. During the years 1990-1992, 14,541 pregnant women residing in Avon, Great Britain with an estimated delivery date between April 1991 and December 1992 were enrolled in the study. These (initial) pregnancies resulted in a total of 14,062 live births and 13,988 children alive at one year of age. An additional 713 eligible children were enrolled at approximately 7 years of age and their data are available for analyses when including variables collected from the age of seven and later. Details of recruitment methods are described in detail elsewhere (Fraser et al., 2013; Boyd et al., 2013).

This study examined associations between maternal exposure to PCBs and fetal growth in girls in an ancillary study designed to look at the association between maternal serum concentrations of environmental exposures and daughter's puberty characteristics (Christensen et al., 2011). To be considered, girls had to have at least two pubertal assessments to allow for classification of age at menarche. The ancillary study included all girls with early menarche ( $<11.5$ years; $n=218)$ and a random sample of girls without early menarche $\geq 11.5$ years $(n=230)$. Informed consent was provided at the time of enrollment by the mothers. Human subjects' protection and ethical approval were provided by the ALSPAC Law and Ethics Committee, the Local Research Ethics Committees, and the Centers for Disease Control and Prevention (CDC) Institutional Review Board. Please note that the study website contains details of all the data that are available through a fully searchable data dictionary, http://www.bris.ac.uk/alspac/researchers/data-access/datadictionary.

\subsection{Data collection}

Outcomes of interest included birth weight (in grams), birth length (in centimeters), and gestational age (in weeks) which were abstracted from medical records. Low birth weight was considered $<2500 \mathrm{~g}$. Ponderal index was calculated using the following formula: (weight in $\mathrm{g} /$ height in $\mathrm{cm}^{3}$ ) $\times 100$ (Maisonet et al., 2012). Self-reported data on maternal prenatal characteristics and behaviors were obtained from questionnaires completed during 
pregnancy. Data collection and methods have been described in detail elsewhere (Golding et al., 2001).

\subsection{Laboratory analyses}

Blood samples were collected during pregnancy, processed and serum was stored frozen at $20{ }^{\circ} \mathrm{C}$. In 2008, samples were shipped to the National Center for Environmental Health's Division of Laboratory Sciences, Centers for Disease Control and Prevention where PCB-118, PCB-153 and PCB-187 were measured by gas chromatography isotope dilution high resolution mass spectrometry (GC-IDHRMS) (Hovander et al., 2000). This analysis presents lipid-adjusted exposures and measurements recorded as $0 \mathrm{ng} / \mathrm{g}$ are noted as < LOD.

\subsection{Statistical analyses}

Pearson correlation coefficients were used to assess the relationship between PCB analytes. Stratum-weighted linear models, which accounted for the nested case-control study design, were used to estimate the association between individual PCB concentrations (PCB-118, PCB-153, and PCB-187) and fetal growth markers (birth weight, birth length, gestational age, and ponderal index). To adjust for the original selection criteria for the nested casecontrol study, cases (all girls who attained menarche $<11.5$ years) and controls (girls who attained menarche $\geq 11.5$ years) were assigned weights of 1 and 15.1, respectively. Each PCB analyte was examined individually in a stratum-weighted model for each birth outcome, and backwards elimination was used to identify potential covariates which appreciably contributed to model fit or interpretation. Covariates considered included previous births ( $0 /$ 21), maternal age (continuous), maternal race (white/non-white), pre-pregnancy BMI (continuous), educational status (categorical), tobacco use (binary), and gestational age when maternal serum sample was obtained (continuous). In this analysis, not attaining any General Certificates of Secondary Education (GCSEs, at 16 years of age) was coded as " < O" (low) educational level, obtaining GCSEs as "O" (medium) and completing GCSEs and/or vocational training with additional education (e.g., University or Advanced) was considered " > O level" (high). After backwards elimination, the remaining covariates for birth weight outcome included previous births, maternal BMI, race, education, tobacco use during pregnancy and gestational ages at sample collection. For birth length, the confounders included in the model were parity and maternal BMI. The ponderal index model included the confounders, parity, maternal BMI, and tobacco use during pregnancy. For gestational age at birth, the only confounder remaining in the model was gestational age when serum sample was collected. Effect modification by maternal smoking and maternal education was also examined by testing appropriate interaction terms for statistical significance. SAS version 9.3 (SAS Institute Inc., Cary, NC) was used to conduct all analyses. All statistical tests were 2-tailed; a $p$-value of $<0.05$ was considered statistically significant.

\section{Results}

More than half of the mothers were 29 years of age or younger at delivery and reported having normal pre-pregnancy BMI. About half of the mothers had an educational level of less than or equal to O level and most mothers were white (94\%) (Table 1). The median 
gestational age at serum sample collection was 15 weeks with an interquartile range of 1028 weeks. Among 448 daughters included in the study population, mean birth weight ( \pm $\mathrm{SD})$ was $3396.7( \pm 498.7) \mathrm{g}$; birth length was $50.4( \pm 2.2) \mathrm{cm}$; ponderal index was 2.7 $( \pm 0.2) \mathrm{kg} / \mathrm{m}^{3}$; and gestational age was $39.8( \pm 1.6)$ weeks (data not presented). Fewer than $4 \%$ of daughters were born preterm or weighed $<2500 \mathrm{~g}$.

PCB analytes were detected in > 98\% of the samples tested. PCB-153 had the highest median maternal serum concentrations followed by PCB-118 and PCB-187 (Table 1). Median (min-max) concentration in maternal samples of PCB-153 was 64.5 (3.7-200.1) ng/g; PCB-118 was 14.9 (< LOD-90.9) ng/g; and PCB-187 was 11.3 (< LOD-41.6) ng/g. Pearson correlation coefficients showed high levels of correlation between PCB-118 and PCB-153 $(r=0.80)$ and PCB-118 and PCB-187 $(r=0.73)$, and a very high level of correlation between PCB-153 and PCB-187 $(r=0.90)$.

Birth length, ponderal index and gestational age were not associated with any of the PCB analytes in main effects' models (Table 2). There was significant effect modification between maternal education and PCB analytes (PCB-118, PCB-153, and PCB-187) for birth weight; therefore, results for birth weight were stratified by maternal education (Table 3 ). For all three analytes, we observed significant inverse associations with birth weight only among daughters of mothers with less than O-level (low) education. Daughter's birth weight was $-138.4 \mathrm{~g}$ lower (95\% CI: -218.0, -58.9) for each $10 \mathrm{ng} / \mathrm{g}$ lipid increase in maternal serum PCB-118. Similarly, every $10 \mathrm{ng} / \mathrm{g}$ lipids increase in maternal serum PCB-153 was associated with a $-41.9 \mathrm{~g}(95 \% \mathrm{CI}:-71.6,-12.2)$ lower birth weight. Every $10 \mathrm{ng} / \mathrm{g}$ lipids increase in maternal serum PCB-187, was associated with a $-170.4 \mathrm{~g}$ (95\% CI: -306.1, -34.7) lower birth weight (Table 3), among girls with mothers in the lowest education group.

\section{Discussion}

In this analysis, we explored the associations between prenatal exposure to PCB-118, PCB-153 and PCB-187 and fetal growth markers; birth weight, birth length, ponderal index, and gestational age. Our findings suggest that prenatal exposure to PCBs is inversely associated with birth weight and that mother's education significantly modified the association between maternal PCB concentrations and birth weight in female newborns. Educational status may be a marker for socioeconomic status in this population; both education and socioeconomic status have positively associated with maternal diet quality and food security (Rifas-Shiman et al., 2009). This could in part contribute to the differences that we observed by mother's education.

Evidence from previous studies suggests prenatal exposure to PCBs has harmful effects on fetal growth (Kezios et al., 2012; Karmaus and Zhu, 2004; Blanck et al., 2002). A metaanalysis of 12 European cohorts including 7990 mother-child dyads and a pooled study of 9377 mother-child dyads from 11 European cohorts (15 studies) both observed inverse associations between fetal PCB-153 exposure and birth weight (Govarts et al., 2012; Casas et al., 2015). Compared to the maternal serum levels observed in other European studies, our median PCB-153 concentration (64.5 ng/g lipid), while not the lowest observed, does fall in the lower half of overall results (range of median PCB-153 across European studies 15.3- 
394.4 ng/g lipid) (Casas et al., 2015). In contrast, Longnecker et al., in the U.S. Collaborative Perinatal Project of 1034 pregnant women enrolled between 1959 and 1965, found no associations between birth weight or gestational age and serum concentrations of 11 PCB analytes collected during the third trimester of pregnancy (Longnecker et al., 2005). Lignell et al., observed that breast milk concentrations of PCB-138, PCB-153 and PCB-180 assessed within the first month post-delivery were positively associated with birth weight in a sample of first-time mothers in Sweden $(n=411)$ (Lignell et al., 2013) with stronger associations reported for males than females.

Inconsistent findings across studies may be related to multiple factors such as differences in study design, population characteristics, sample sizes, timing or type of sample measured, and overall distribution of exposure (e.g., low v. high). PCB levels measured in breast milk collected after birth may not be representative of in utero exposure. Also, differences in the PCB analytes examined can lead to varied findings since PCB analytes vary in their ability to bioaccumulate and in level of toxicity (Longnecker et al., 2005; McGraw and Waller, 2009). PCBs with lower degrees of chlorination tend to be more rapidly excreted from the body; thus, may be poorer biomarkers of long-term exposure.

Although the possible mechanism of PCBs on birth weight is not known, it could be related to the endocrine-disrupting properties of PCBs (Kaijser et al., 2000). Estrogens promote fetal growth and PCBs have been found to have both estrogenic and anti-estrogenic roles (Hamers et al., 2011). In animal models, a growing body of literature suggests that exposure to endocrine-disrupting chemicals can have a wide range of effects on metabolism such altering insulin metabolism and disrupting energy balance (Ross, 2004; Vandenberg et al., 2012). For example, PCB153 concentrations have been inversely associated with serum thyroxine levels in an animal model (Ness et al., 1993).

Our analysis was conducted on a sample of mothers and their daughters chosen from a nested case-control study for pubertal development. Although our results could be biased if the girls excluded from the analysis were different from girls who were included in the analysis, it is unlikely since mean values of maternal characteristics and fetal growth outcomes for the study sample are similar to those of the group of girls enrolled in the full cohort (Maisonet et al., 2012). These analyses were performed on a sample of girls because data on prenatal exposure to PCBs are not currently available for ALSPAC boys. It is unknown whether these results can be extrapolated to males. Additionally, PCB measurements are only available for a single time point during pregnancy and daughters' PCB concentrations measured at birth are not available. The timing of specimen collection is important and in our analyses we controlled for gestational age at sample collection, because evidence suggests that concentrations decline from preconception to postnatally sensitive windows (Bloom et al., 2007). Further analyses will explore the association between prenatal PCB exposure and postnatal growth in girls at other time points. Lastly, there is the possibility of residual confounding by unmeasured or poorly measured (e.g., maternal smoking) covariates. 


\section{Conclusions}

Our results are consistent with results from other studies that show an association between higher prenatal PCB levels and lower birth weight (Karmaus and Zhu, 2004; Govarts et al., 2012; Murphy et al., 2010). Birth weight is reflective of fetal development from conception to birth, and low birth weight is associated with negative health outcomes later in life. Preterm or low birth weight infants are at a heightened risk for morbidities and chronic conditions, including cardiovascular diseases and adverse behavioral, cognitive and psychiatric outcomes (Givens et al., 2007). In our study, mother's education, a possible marker for socioeconomic status, significantly modified the association between maternal PCB concentrations and birth weight in female newborns. We observed significant inverse associations with birth weight only among daughters of mothers with less education (lower socioeconomic status). This relationship has not previously been identified and should be investigated in future research.

\section{Acknowledgements}

We are extremely grateful to all the families who took part in this study, the midwives for their help in recruiting them, and the whole ALSPAC team, which includes interviewers, computer and laboratory technicians, clerical workers, research scientists, volunteers, managers, receptionists and nurses. The UK Medical Research Council and the Wellcome Trust (Grant ref.: 092731) and the University of Bristol provide core support for ALSPAC. This research was specifically funded by the US Centers for Disease Control and Prevention (CDC). KN is funded by the National Institute for Health Research Collaboration for Leadership in Applied Health Research and Care (NIHR CLAHRC) West at University Hospitals Bristol NHS Foundation Trust. This publication is the work of the authors and they will serve as guarantors for the contents of this paper.

\section{References}

ATSDR, 2000 Public Health Statement Polychlorinated Biphenyls (PCBS).

Barker DJ, 1998 In utero programming of chronic disease. Clin. Sci. (Lond.) 95 (2), 115-128. [PubMed: 9680492]

Barker DJ, 2002 Fetal programming of coronary heart disease. Trends Endocrinol. Metab 13 (9), $364-$ 368. [PubMed: 12367816]

Blanck HM, Marcus M, Rubin C, et al., 2002 Growth in girls exposed in utero and postnatally to polybrominated biphenyls and polychlorinated biphenyls. Epidemiology 13 (2), 205-210. [PubMed: 11880762]

Bloom MS, Buck Louis GM, Schisterman EF, et al., 2007 Maternal serum polychlorinated biphenyl concentrations across critical windows of human development. Environ. Health Perspect 115 (9), 1320-1324. [PubMed: 17805422]

Boyd A, Golding J, Macleod J, et al., 2013 Cohort profile: the 'children of the 90s ' - the index offspring of the Avon longitudinal study of parents and children. Int. J. Epidemiol 42 (1), 111-127. [PubMed: 22507743]

Casas M, Nieuwenhuijsen M, Martinez D, et al., 2015 Prenatal exposure to PCB-153, p,p'-DDE and birth outcomes in 9000 mother-child pairs: exposure-response relationship and effect modifiers. Environ. Int 74, 23-31. [PubMed: 25314142]

Christensen KY, Maisonet M, Rubin C, et al., 2011 Exposure to polyfluoroalkyl chemicals during pregnancy is not associated with offspring age at menarche in a contemporary British cohort. Environ. Int 37 (1), 129-135. [PubMed: 20843552]

El Majidi N, Bouchard M, Gosselin NH, et al., 2012 Relationship between prenatal exposure to polychlorinated biphenyls and birth weight: a systematic analysis of published epidemiological studies through a standardization of biomonitoring data. Regul. Toxicol. Pharmacol 64 (1), 161176. [PubMed: 22735367] 
Fraser A, Macdonald-Wallis C, Tilling K, et al., 2013 Cohort profile: the Avon longitudinal study of parents and children: ALSPAC mothers cohort. Int. J. Epidemiol 42 (1), 97-110. [PubMed: 22507742]

Givens ML, Small CM, Terrell ML, et al., 2007 Maternal exposure to polybrominated and polychlorinated biphenyls: infant birth weight and gestational age. Chemosphere 69 (8), 12951304. [PubMed: 17617441]

Golding J, Pembrey M, Jones R, 2001 ALSPAC - the Avon longitudinal study of parents and children. I. Study methodology. Paediatr. Perinat. Epidemiol 15 (1), 74-87. [PubMed: 11237119]

Govarts E, Nieuwenhuijsen M, Schoeters G, et al., 2012 Birth weight and prenatal exposure to polychlorinated biphenyls (PCBs) and dichlorodiphenyldichloroethylene (DDE): a meta-analysis within 12 European birth cohorts. Environ. Health Perspect 120 (2), 162-170. [PubMed: 21997443]

Hamers T, Kamstra JH, Cenijn PH, et al., 2011 In vitro toxicity profiling of ultrapure non-dioxin-like polychlorinated biphenyl congeners and their relative toxic contribution to PCB mixtures in humans. Toxicol. Sci 121 (1), 88-100. [PubMed: 21357386]

Hovander L, Athanasiadou M, Asplund L, et al., 2000 Extraction and cleanup methods for analysis of phenolic and neutral organohalogens in plasma. J. Anal. Toxicol 24 (8), 696-703. [PubMed: 11110024]

Jacobson JL, Fein GG, Jacobson SW, et al., 1984 The transfer of polychlorinated biphenyls (PCBs) and polybrominated biphenyls (PBBs) across the human placenta and into maternal milk. Am. J. Public Health 74 (4), 378-379. [PubMed: 6322600]

Kaijser M, Granath F, Jacobsen G, et al., 2000 Maternal pregnancy estriol levels in relation to anamnestic and fetal anthropometric data. Epidemiology 11 (3), 315-319. [PubMed: 10784250]

Karmaus W, Zhu X, 2004 Maternal concentration of polychlorinated biphenyls and dichlorodiphenyl dichlorethylene and birth weight in Michigan fish eaters: a cohort study. Environ. Health 3 (1), 1. [PubMed: 14748928]

Kezios KL, Liu X, Cirillio PM, et al., 2012 Prenatal polychlorinated biphenyl exposure is associated with decreased gestational length but not birth weight: archived samples from the Child Health and Development Studies pregnancy cohort. Environ. Health 11, 49. [PubMed: 22817616]

Koopman-Esseboom C, Morse DC, Weisglas-Kuperus N, et al., 1994 Effects of dioxins and polychlorinated biphenyls on thyroid hormone status of pregnant women and their infants. Pediatr. Res 36 (4), 468-473. [PubMed: 7816522]

Law CM, Shiell AW, Newsome CA, et al., 2002 Fetal, infant, and childhood growth and adult blood pressure: a longitudinal study from birth to 22 years of age. Circulation 105 (9), 1088-1092. [PubMed: 11877360]

Lignell S, Aune M, Darnerud PO, et al., 2013 Prenatal exposure to polychlorinated biphenyls (PCBs) and polybrominated diphenyl ethers (PBDEs) may influence birth weight among infants in a Swedish cohort with background exposure: a cross-sectional study. Environ. Health 12, 44. [PubMed: 23724965]

Longnecker MP, Klebanoff MA, Brock JW, et al., 2005 Maternal levels of polychlorinated biphenyls in relation to preterm and small-for-gestational-age birth. Epidemiology 16 (5), 641-647. [PubMed: 16135940]

Maisonet M, Terrell ML, McGeehin MA, et al., 2012 Maternal concentrations of polyfluoroalkyl compounds during pregnancy and fetal and postnatal growth in British girls. Environ. Health Perspect 120 (10), 1432-1437. [PubMed: 22935244]

McGraw JE, Waller DP, 2009 Fish ingestion and congener specific polychlorinated biphenyl and p,p'dichlorodiphenyldichloroethylene serum concentrations in a great lakes cohort of pregnant African American women. Environ. Int 35 (3), 557-565. [PubMed: 19046769]

Murphy LE, Gollenberg AL, Buck Louis GM, et al., 2010 Maternal serum preconception polychlorinated biphenyl concentrations and infant birth weight. Environ. Health Perspect 118 (2), 297-302. [PubMed: 20123616]

Ness DK, Schantz SL, Moshtaghian J, et al., 1993 Effects of perinatal exposure to specific PCB congeners on thyroid hormone concentrations and thyroid histology in the rat. Toxicol. Lett 68 (3), 311-323. [PubMed: 8516785] 
Patandin S, Koopman-Esseboom C, de Ridder MA, et al., 1998 Effects of environmental exposure to polychlorinated biphenyls and dioxins on birth size and growth in Dutch children. Pediatr. Res 44 (4), 538-545. [PubMed: 9773843]

Rifas-Shiman SL, Rich-Edwards JW, Kleinman KP, et al., 2009 Dietary quality during pregnancy varies by maternal characteristics in Project Viva: a US cohort. J. Am. Diet. Assoc 109 (6), 10041011. [PubMed: 19465182]

Ritter R, Scheringer M, MacLeod M, et al., 2011 Intrinsic human elimination half-lives of polychlorinated biphenyls derived from the temporal evolution of crosssectional biomonitoring data from the United Kingdom. Environ. Health Perspect 119 (2), 225-231. [PubMed: 20934951]

Ross G, 2004 The public health implications of polychlorinated biphenyls (PCBs) in the environment. Ecotoxicol. Environ. Saf 59 (3), 275-291. [PubMed: 15388267]

Taylor PR, Stelma JM, Lawrence CE, 1989 The relation of polychlorinated biphenyls to birth weight and gestational age in the offspring of occupationally exposed mothers. Am. J. Epidemiol 129 (2), 395-406. [PubMed: 2492144]

Thompson C, Syddall H, Rodin I, et al., 2001 Birth weight and the risk of depressive disorder in late life. Br. J. Psychiatry 179, 450-455. [PubMed: 11689404]

Vandenberg LN, Colborn T, Hayes TB, et al., 2012 Hormones and endocrine-disrupting chemicals: low-dose effects and nonmonotonic dose responses. Endocr. Rev 33 (3), 378-455. [PubMed: 22419778]

WHO, 1992 Low Birth Weight. 92 pp. 2.

Wohlfahrt-Veje C, Audouze K, Brunak S, et al., 2014 Polychlorinated dibenzo-p-dioxins, furans, and biphenyls (PCDDs/PCDFs and PCBs) in breast milk and early childhood growth and IGF1. Reproduction 147 (4), 391-399. [PubMed: 24586095] 


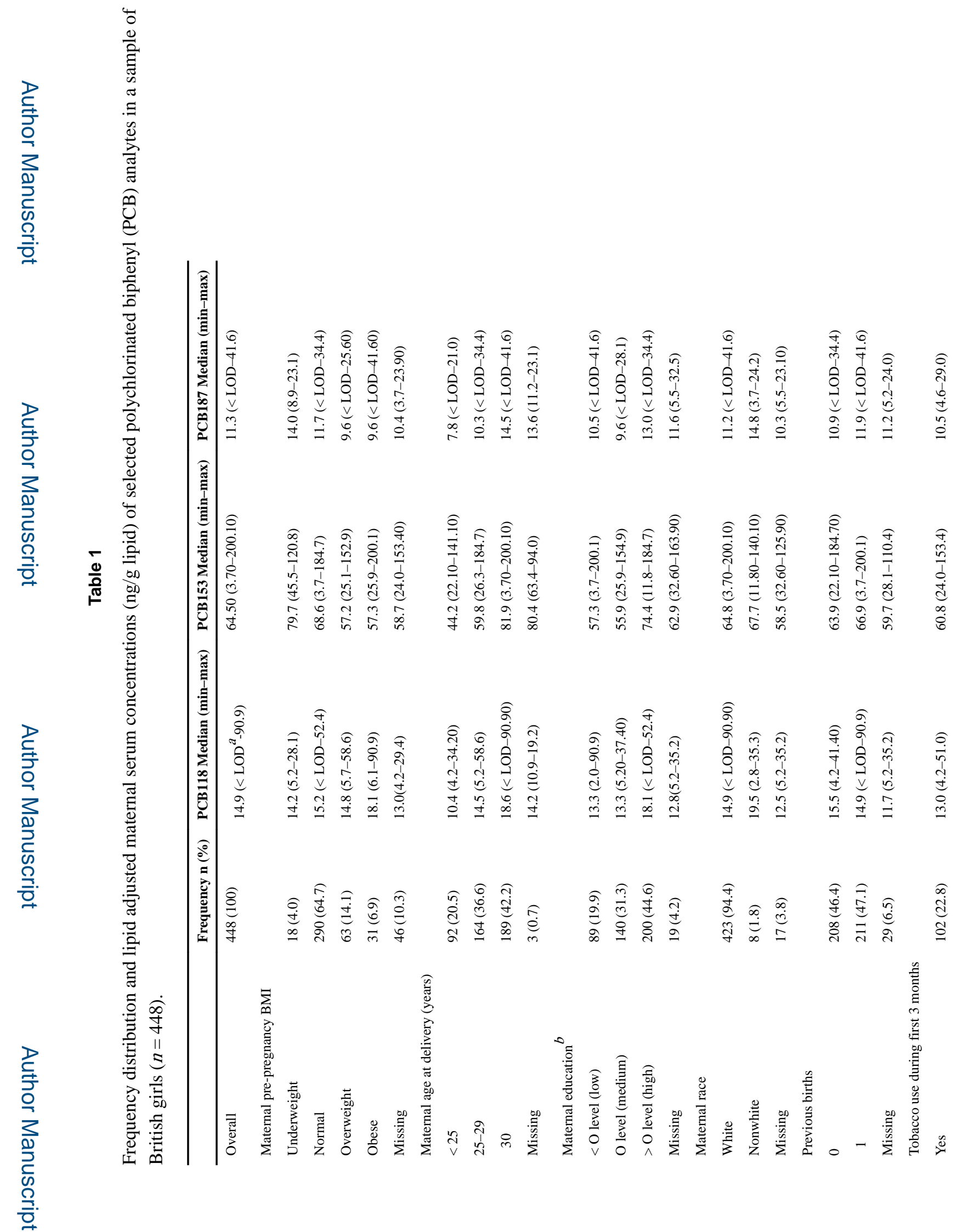

Environ Int. Author manuscript; available in PMC 2019 July 01. 
Patel et al.

Page 11

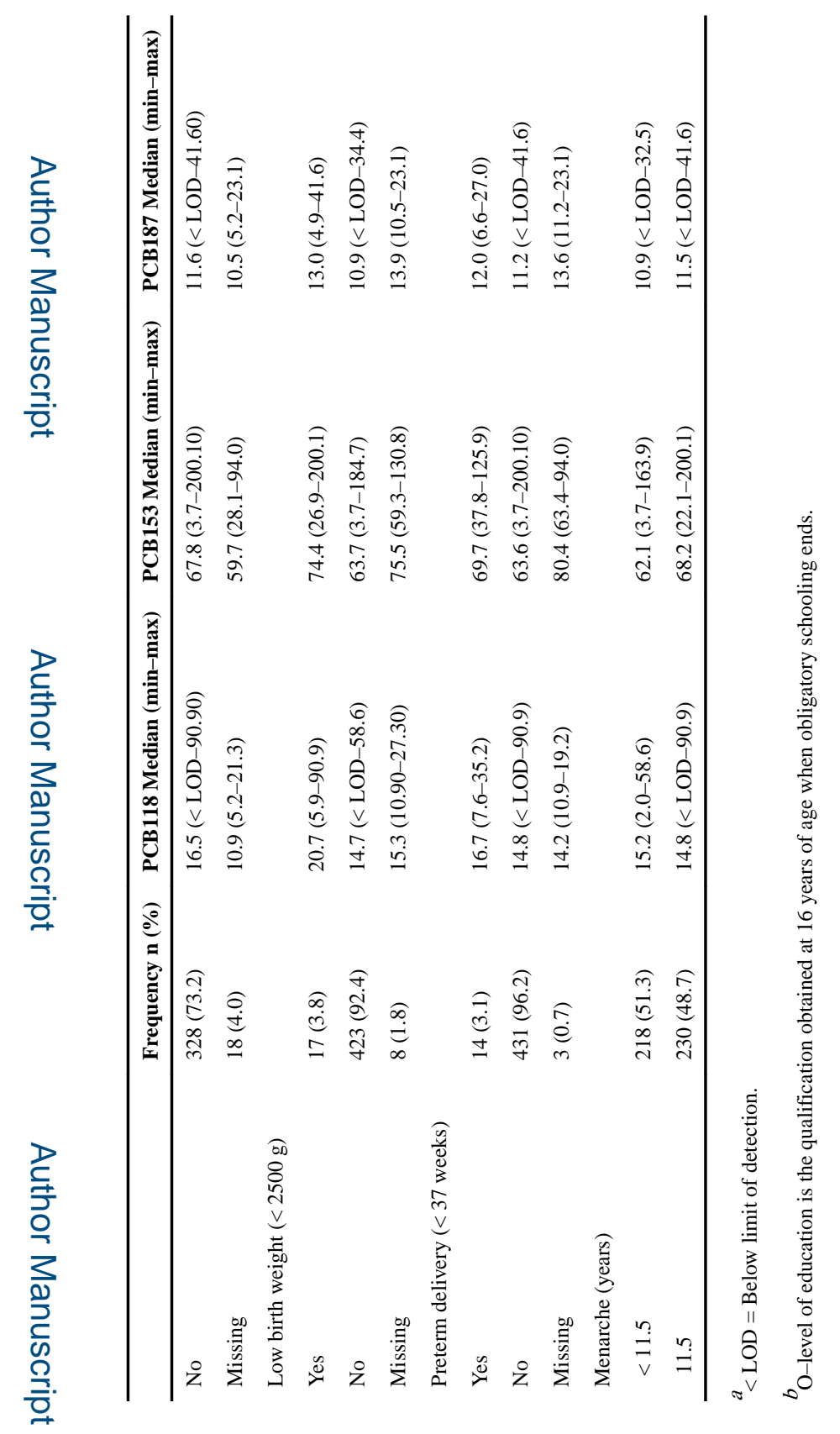

로을

Environ Int. Author manuscript; available in PMC 2019 July 01. 
Patel et al.

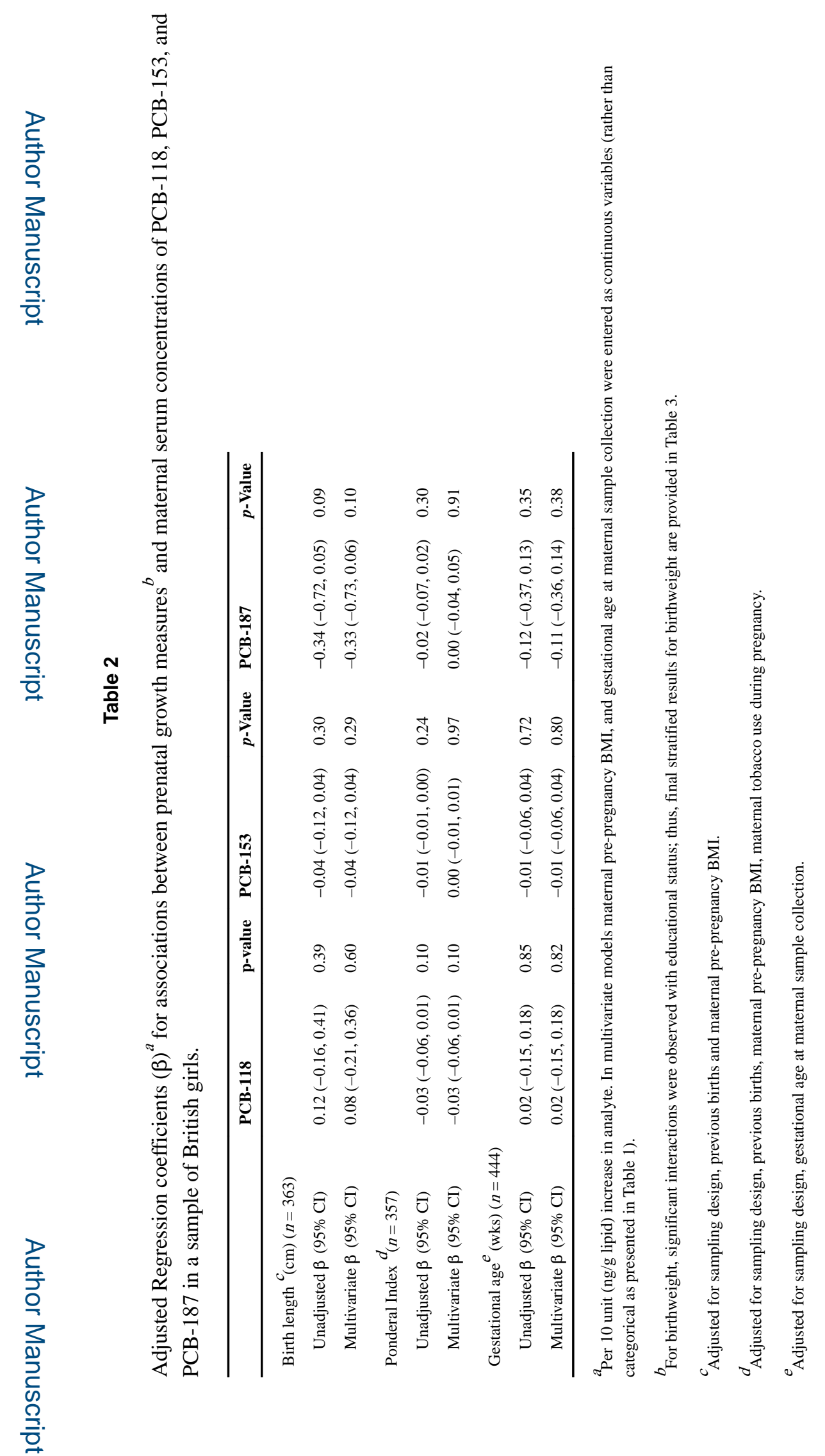

Environ Int. Author manuscript; available in PMC 2019 July 01. 


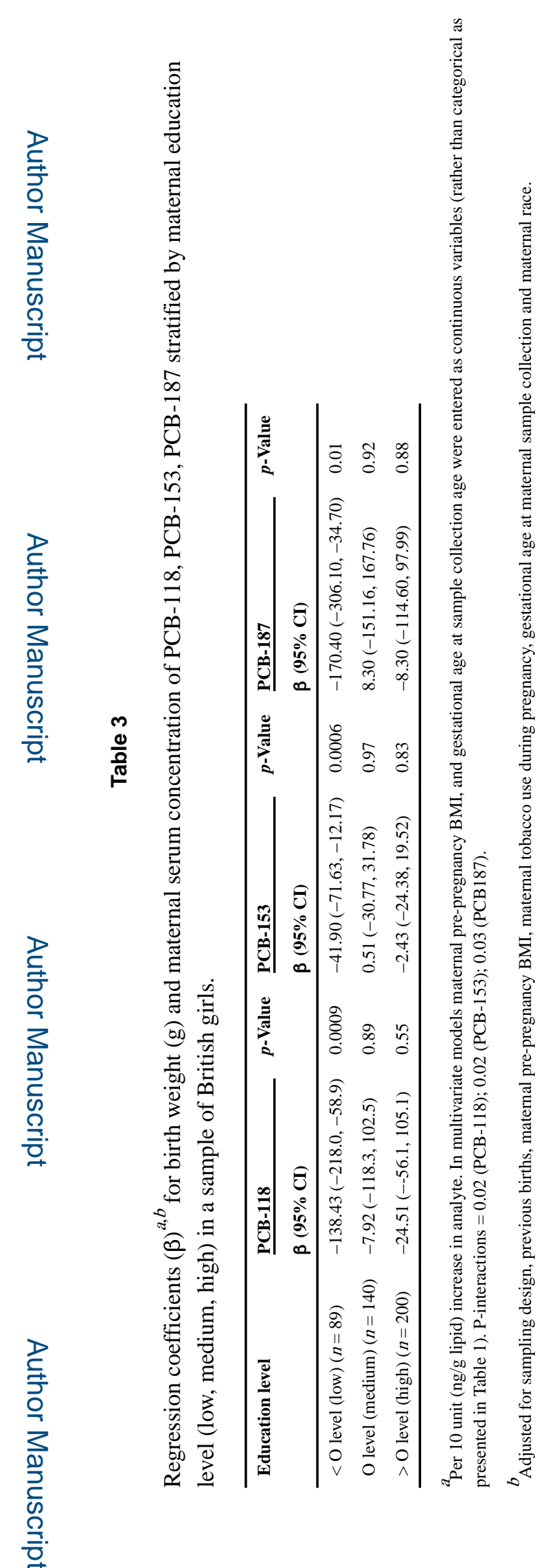

Environ Int. Author manuscript; available in PMC 2019 July 01. 